\title{
Psicología y valoración de la dependencia
}

\section{Psychology and valuation of the dependency}

\section{Ana Maria MÉNDEZ PARDO*}

\section{RESUMEN}

La valoración de la Dependencia en el marco de la futura Ley de Protección, conlleva la necesidad de creación de un Instrumento adecuado a tal finalidad. A lo largo de este trabajo se definen las características que habrá de tener el mismo y el proceso que se está siguiendo en su elaboración. Finalmente se exponen como se contemplarán los aspectos psicológicos de la persona en tal valoración.

\section{PALABRAS CLAVE}

Dependencia, Baremo, Actividades, Aspectos psicológicos.

\section{ABSTRACT}

The valuation of the Dependency within the framework of the future Law of Protection entails the necessity of creation of an Instrument adapted to such purpose. Throughout this work the characteristics needed and the process being followed in its elaboration are defined. Finally the way to contemplate the psychological aspects of the person in such valuation will be exposed.

\section{KEY WORDS}

Dependency, Scale, Activities, Psychological aspects.

\footnotetext{
* Psicóloga. Técnico de la Dirección General de Politicas Sociales. Consejería de Sanidad y Servicios Sociales del Gobierno de Cantabria. Colaboradora con el Instituto del Envejecimiento de la Universidad Autónoma de Barcelona (FICE).
} 


\section{INTRODUCCIÓN}

La creación de un sistema de protección de las personas en situación de dependencia en el estado español constituye uno de los asuntos básicos de la política social en los próximos años. El reconocimiento de los derechos sociales como derechos fundamentales, y como tales universales y subjetivos, supone un paso cuantitativo y cualitativo fundamental para el Estado de Bienestar. La Ley que actualmente se debate en el Parlamento nos ofrece, también a nosotros, como profesionales, la oportunidad de mejorar la calidad de nuestro sistema de protección social.

A lo largo de esta exposición, me referiré a las características y el objetivo que establece el Proyecto de Ley para el baremo que servirá, en el marco de la futura Ley, para la valoración de los diferentes grados y niveles de dependencia. A continuación describiré el proceso que se está llevando a cabo en la elaboración de la propuesta de este baremo. Explicaré brevemente, algunas características generales del citado instrumento de valoración. Por último expondré como se contemplan en él los aspectos psicológicos de la persona.

\section{IMPORTANCIA DE UN BAREMO PARA LA VALORACIÓN DE LA SITUACIÓN DE DEPENDENCIA}

El Proyecto de Ley de Promoción de la autonomía Personal y Atención a las Personas en situación de Dependencia, establece que "el grado y niveles de dependencia, a efectos de su valoración, se determinarán mediante la aplicación del baremo que se acuerde en el Consejo territorial del Sistema Nacional de Dependencia para su posterior aprobación por el Gobierno mediante Real Decreto" (Art. 27)
El Baremo se puede considerar la puerta de entrada en el sistema de protección. Es un instrumento técnico básico para definir cuales van a ser las personas a atender y con que nivel de atención. No se trata de un instrumento destinado a la realización de un plan integral de atención, para lo que los profesionales se deberán de dotar de otros instrumentos complementarios, que se utilizan habitualmente en nuestra práctica profesional.

El documento en el que actualmente se trabaja constituye una propuesta, que deberá elevarse al Consejo Territorial del Sistema Nacional de Dependencia, para su posterior aprobación, modificación o sustitución por otro baremo que se considere más idóneo. El baremo finalmente acordado, será aprobado por el Gobierno, y publicado mediante Real Decreto.

Posteriormente, serán las Comunidades Autónomas, las que determinarán cuales serán los órganos de valoración que emitan el dictamen sobre el grado y nivel de dependencia de la persona, utilizando para ello el baremo aprobado, y los cuidados que esta puede necesitar. Serán igualmente competentes para gestionar los servicios y recursos necesarios para la valoración de la dependencia.

Tendremos por tanto, un baremo único para todo el Estado, de manera que el reconocimiento de grado y nivel de dependencia en una Comunidad Autónoma será válido en cualquier otra. El baremo, aunque podrá ser aplicado por equipos interdisciplinares compuestos por diferentes profesionales a criterio de cada Comunidad, deberá de ajustarse a los procedimientos y criterios técnicos, que apruebe el Consejo Territorial del Sistema Nacional de Dependencia. Ambas condiciones servirán para garan- 
tizar a todos los ciudadanos del Estado la igualdad en el acceso al Sistema Nacional de Dependencia.

\section{CARACTERÍSTICAS GENERALES DEL BAREMO}

El Proyecto de Ley establece las características que tendrá el baremo:

- Habrá de tener entre sus referentes la Clasificación Internacional del Funcionamiento, la Discapacidad y la Salud (CIF), aprobada por la OMS en el año 2001.

- Establecerá los criterios objetivos de valoración del grado de autonomía de la persona y de su capacidad para realizar las distintas actividades de la vida diaria.

- Cifrará los intervalos de puntuación para cada uno de los grados y niveles de dependencia.

- Contará con un protocolo y técnicas a seguir para la valoración de las aptitudes observadas

\section{OBJETO DE LA VALORACIÓN}

Es importante recordar la definición de lo que es la Dependencia que ofrece el Proyecto de Ley: "El estado de carácter permanente en que se encuentran las personas que, por razones derivadas de la edad, la enfermedad o la discapacidad, y ligadas a la falta 0 a la pérdida de autonomia física, mental, intelectual o sensorial, precisan de la atención de otra $u$ otras personas $o$ ayudas importantes para realizar las actividades básicas de la vida diaria”.

El cuerpo básico del baremo será la Valoración de la Situación de Depen- dencia, en función de la autonomía de las persona y de la necesidad de cuidados que esta requiere.

El objetivo fundamental habrá de ser el análisis de la capacidad de la persona para realizar por sí misma las Actividades Básicas de la Vida Diaria (ABVD). El Proyecto de Ley, define a estas como "las tareas más elementales de la persona, que le permiten desenvolverse con un minimo de independencia, tales como: el cuidado personal, las actividades domésticas básicas, la movilidad esencial, reconocer personas y objetos, orientarse, entender y ejecutar órdenes o tareas sencillas". Siguiendo los criterios de la Clasificación Internacional del Funcionamiento, la Discapacidad y la Salud, las dificultades que una persona puede tener para realizar estas actividades pueden aparecer como una alteración cualitativa o cuantitativa en la manera en que la persona desempeña la actividad en comparación con otras que no tienen un problema de salud similar.

Dado que la situación de dependencia se clasifica en tres grados (Dependencia moderada, dependencia severa y gran dependencia), según cual sea la intensidad y frecuencia de la ayuda que la persona precisa para realizar las actividades, un aspecto fundamental en la valoración es la relativa a esta necesidad de ayuda. El baremo calificará, una vez establecida la necesidad, el grado de apoyo de otra persona que esta precisa.

El mismo Proyecto de Ley recoge las condiciones en que deberá ser valorada la situación de Dependencia de la persona en la realización de estas Actividades de la Vida Diaria:

- En el entorno habitual en el que vive la persona. 
La propuesta en la que se trabaja está siendo diseñada para ser aplicada en el domicilio habitual.

- Teniendo en cuenta las ayudas técnicas, ortesis y prótesis prescritas y disponibles.

La valoración se realizará utilizando la persona aquellas de las que dispone.

- Considerando las barreras existentes en el entorno.

Esto nos remite al concepto desempeño/realización, de la Clasificación Internacional del Funcionamiento, la Discapacidad y la Salud de la OMS. Se trata de describir lo que la persona hace en el contexto/entorno actual y real en el que vive. El contexto/entorno actual también se describe utilizando los Factores Ambientales, de los que forman parte las Ayudas técnicas y barreras. Es un concepto importante pues puede darse la situación de que personas con capacidades similares y en entornos distintos puedan presentar niveles de dependencia diversos.

La propuesta de baremo que se está testando en estos momentos se complementa con dos informes. El primero de ellos relativo a las Condiciones de Salud. Se trata de un Informe sobre las enfermedades y deficiencias, de carácter permanente que padece la persona, y que son las determinantes de la situación de dependencia.

Recordemos la definición que la Clasificación Internacional del Funcionamiento, la Discapacidad y la Salud (CIF), de la OMS da del término Deficiencias: "Son problemas en las funciones fisiológicas o en las estructuras corporales de una persona. Pueden consistir en una pérdida, defecto, anomalía o cualquier otra desviación significativa respecto a la norma estadísticamente establecida”.

Este informe es fundamental para facilitar la valoración y comprender mejor la situación de la persona por parte del personal valorador.

El segundo informe al que nos referíamos antes, incluirá Información complementaria, con la finalidad de reunir un conjunto mínimo de datos que, en la propuesta en la que se sigue trabajando, se refieren a las características del entorno domiciliario habitual de la persona, empleo de ayudas técnicas y realización de tareas domésticas básicas. Los datos recogidos en este informe no influirán en la valoración de la severidad de la dependencia, pero pueden ser útiles para orientar en la realización del Programa Individual de Atención.

El proceso de valoración finalizará, con la elaboración del Programa Individual de Atención, en el que se determinarán tras la consulta a la persona interesada, y, en su caso, a su familia o entidades tutelares que le representen, las modalidades de intervención más adecuadas (servicios 0 prestaciones) a sus necesidades.

\section{PROCESO DE ELABORACIÓN DEL BAREMO}

La elaboración de una propuesta de este baremo fue encargada por el Gobierno, a través del IMSERSO, al Instituto del Envejecimiento de la Universidad Autónoma de Barcelona, (en adelante FICE).

Entre el 30 de mayo y el 10 de junio de 2005, el FICE sometió a consideración de un panel Delphy de expertos un primer cuestionario de 41 preguntas, en cada una de las cuales se abordaba un aspecto del baremo. Este grupo de expertos estaba formado por 25 personas de 
diferentes profesiones (5 de ellas psicólogos) con conocimientos y experiencia en envejecimiento y discapacidad, además de representantes de diferentes comunidades Autónomas y Entidades representantes de colectivos.

En esta primera consulta se trabajó sobre una propuesta de baremo para la Valoración de la Dependencia en la que, además de los aspectos generales relativos a las instrucciones y condiciones de aplicación del mismo, se intentó identificar un consenso sobre las actividades y tareas incluidas en el mismo y explorar el peso específico a asignar a cada actividad y dentro de la misma a las diferentes tareas. Del mismo modo se buscó un acuerdo sobre la calificación y definición de los apoyos y ayudas que la persona puede precisar.

Del 4 al 15 de julio de 2005, se llevó a cabo la segunda parte del panel Delphy. Tras haber logrado un consenso superior al $80 \%$ en 22 de las 41 cuestiones planteadas en la primera parte, se sometieron de nuevo a consideración 14 cuestiones.

Técnicos del FICE aplicaron el baremo para la Valoración de la Dependencia a una muestra de 250 personas.

Durante todo el año 2006 se ha continuado trabajando en la adaptación y mejora de algunos aspectos del instrumento: adaptación del mismo para su aplicación en niños de tres y más años; instrucciones relativas a la aplicación en personas con retraso mental y trastorno mental y consenso sobre el informe en el que se recogen aspectos relativos a las condiciones de salud. Así mismo el FICE ha elaborado un manual para los valoradores al objeto de establecer criterios únicos en la aplicación del baremo y se continúa trabajando en el mismo.

A lo largo del primer semestre de 2006 se mantuvieron diferentes reuniones con los representantes de las Comunidades Autónomas y se les entregó un borrador del baremo para la valoración de la dependencia. En ellas se tomó la decisión de aplicar el instrumento a una muestra nacional significativa para afinar su eficacia en grupos especificos, como las personas con enfermedades mentales o los niños.

El 3 de Julio de 2006 se llevó a cabo una primera reunión de FICE, IMSERSO y Coordinadores designados por las Comunidades Autónomas para la aplicación del pilotaje, a una muestra nacional, de 2.500 personas, formada por 46 de cada provincia del Estado, seleccionadas atendiendo a criterios de edades y deficiencias.

Del 26 al 28 de Julio se impartió el primer curso de formación, de 20 horas de duración, para los valoradores designados por las Comunidades Autónomas a efectos de llevar a cabo el pilotaje. A lo largo de estas jornadas se continuó trabajando en la mejora del Manual de uso para valoradores.

Entre el 4 y el 22 de Septiembre, se realizó la primera aplicación experimental del baremo a la muestra seleccionada en cada provincia del Estado. Se aplicó a las personas que componian la muestra, no solo el baremo de Valoración de la Dependencia, sino también el Baremo de Ayuda de Tercera Persona, el MEC de Lobo y la escala de Barthel.

En estos momentos se trabaja analizando los resultados.

\section{LOS ASPECTOS PSICOLÓGICOS DE LA PERSONA EN LA VALORACIÓN DE LA SITUACIÓN DE DEPENDENCIA}

Volviendo a la definición de la Depen- 
dencia, recordemos que en ella se hace referencia a la pérdida de autonomía fisica, mental, intelectual o sensorial, se consideran por tanto objeto de protección todas las deficiencias, permanentes, relacionadas con funciones mentales.

En estos momentos hay un movimiento de Entidades y Asociaciones de familiares de personas con discapacidad intelectual en el que se critica que los baremos de entrada en el Sistema Nacional de Atención a la Dependencia contemplarán, fundamentalmente, las limitaciones físicas y de movilidad, pero no otras variables que hacen que las personas con discapacidad intelectual precisen apoyos para desarrollar sus vidas de forma autónoma.

A mi modo de ver los profesionales de la Psicologia, y todos los que hemos colaborado en la elaboración de esta propuesta de baremo, estamos haciendo un gran esfuerzo para que se valoren, no solo las dificultades físicas, sino también las cognitivas, de autodeterminación personal, así como las dificultades de tipo conductual, de comprensión del entorno social, etc. que impidan desarrollar las actividades más básicas.

Algunas Actividades Básicas de la Vida Diaria de las recogidas en la definición que de las mismas hace el Proyecto de Ley, tales como entender y ejecutar ordenes o tareas sencillas se introducen en el baremo como un componente de las otras actividades. De esta manera a lo largo de todo el baremo, son múltiples las aclaraciones y puntualizaciones. Como ejemplos, veamos los siguientes:

- En las instrucciones se insistirá en que para valorar la capacidad de la persona para realizar por sí misma y de forma adecuada las actividades y las tareas que se describen, debe tenerse en cuenta tanto su capacidad de ejecución física, como su capacidad mental y/o de iniciativa.

- Cuando se valore la necesidad de ayuda que la persona necesita para realizar las tareas, se contemplará, además de la ayuda física, un tipo de "asistencia especial", que es el que puede precisar esta cuando presenta trastornos del comportamiento que dificultan la prestación de apoyo para la realización de la actividad.

- En las actividades de autocuidado, se valorarán de manera específica, la capacidad para comprender e indicar la necesidad de llevar a cabo las mismas.

- En las actividades de movilidad, cuando se valoren los desplazamientos se considerarán aspectos relativos a la orientación y a la capacidad para evitar correr riesgos y peligros.

Por último, quiero referirme al tratamiento que la futura Ley dará a la protección de los niños y niñas. El Proyecto de Ley, actualmente en fase de tramitación parlamentaria, contempla, fuera del texto articulado y en la Disposición Adicional decimotercera, la protección a los menores de tres años. Así dice específicamente : " Sin perjuicio de los servicios establecidos en los ámbitos educativo y sanitario, el Sistema Nacional de la Dependencia atenderá las necesidades de ayuda a domicilio y, en su caso, prestaciones económicas vinculadas y para cuidados en el entorno familiar a favor de los menores de tres años que presenten graves discapacidades. El Instrumento de valoración previsto en el Artículo 27 de esta Ley incorporará a estos efectos una escala de valoración específica". 
Ana María Méndez Pardo

El equipo de trabajo del Gobierno de Cantabria, al que pertenezco, junto con la Universidad de Salamanca trabajamos en este momento en la elaboración de una propuesta del citado Instrumento para la Valoración de la situación de Dependencia en niños y niñas menores de tres años. El trabajo esta en sus fases iniciales, y es pronto para poder hacer ni tan siquiera una primera presentación del mismo. En relación al baremo general al que nos venimos refiriendo, hemos colaborado en la adaptación del mismo para su aplicación en niños y niñas mayores de tres. Se han seleccionado los ítems a aplicar en diferentes edades atendiendo a criterios de desarrollo evolutivo. 